\title{
CONDITIONS FOR THE UNIFORM CONVERGENCE IN PROBABILITY OF WAVELET DECOMPOSITIONS FOR STOCHASTIC PROCESSES FROM THE SPACE $\operatorname{Exp}_{\varphi}(\Omega)$
}

UDC 519.21

\author{
YU. V. KOZACHENKO AND O. V. POLOS'MAK
}

\begin{abstract}
Conditions for the uniform convergence in probability on the interval $[0, T]$ of wavelet decompositions of Orlicz stochastic processes of exponential type are found in the paper.
\end{abstract}

\section{INTRODUCTION}

In this paper we present the conditions for the uniform convergence in probability of wavelet decompositions of Orlicz stochastic processes of exponential type. The uniform convergence of wavelet decompositions of nonrandom functions is considered in the book [6]. Some problems related to the uniform convergence with probability one and in probability of wavelet decompositions of stochastic processes are studied in the papers [7, 8, 11, 12] for various spaces of random variables.

We consider a strictly Orlicz stochastic process $X=\{X(t), t \in \mathbb{R}\}$ of exponential type. We derive its wavelet decomposition in terms of continuous wavelet functions:

$$
X(t)=\sum_{k \in \mathbb{Z}} \xi_{0 k} \phi_{0 k}(t)+\sum_{j=0}^{\infty} \sum_{k \in \mathbb{Z}} \eta_{j k} \psi_{j k}(t) .
$$

We find sufficient conditions for the uniform convergence of these decompositions in probability to the original stochastic process $X(t)$. Namely, we find conditions under which

$$
\mathrm{P}\left\{\sup _{0 \leq t \leq T}\left|X(t)-X_{n, k_{j}}(t)\right|>\varepsilon\right\} \rightarrow 0
$$

as $n \rightarrow \infty$ and $k_{j} \rightarrow \infty$ for all $j \in \mathbb{N}_{0}:=\{0,1, \ldots\}$, where

$$
X_{n, k_{j}}(t)=\sum_{|k| \leq k_{0}} \xi_{0 k} \phi_{0 k}(t)+\sum_{j=0}^{n-1} \sum_{|k| \leq k_{j}} \eta_{j k} \psi_{j k}(t) .
$$

The paper consists of four sections. Section 2 contains necessary definitions of wavelet analysis and a theorem on the convergence of the wavelet decomposition to the original process in the norm of the space $L_{2}(\mathbb{R})$. In Section 3, we provide some notions of the theory of Orlicz stochastic processes. Section 4 contains conditions for the uniform

2000 Mathematics Subject Classification. Primary 60G12, 42 C40.

Key words and phrases. Stochastic processes, wavelet decomposition, convergence in probability, Orlicz spaces.

This research was supported by the La Trobe University Research Grant \#501821. 
convergence in probability of wavelet decompositions of Orlicz stochastic processes of exponential type.

\section{WAVELET DECOMPOSITIONS OF STOCHASTIC PROCESSES}

Let $\phi=\{\phi(x), x \in \mathbb{R}\}$ and $\psi=\{\psi(x), x \in \mathbb{R}\}$ be two functions of the space $L_{2}(\mathbb{R})$ and let $\widehat{\phi}(y)$ be the Fourier transform of $\phi$ :

$$
\widehat{\phi}(y)=\int_{\mathbb{R}} e^{-i y x} \phi(x) d x .
$$

Assume that

$$
\sum_{k \in \mathbb{Z}}|\widehat{\phi}(y+2 \pi k)|^{2}=1
$$

almost everywhere.

Suppose there exists a periodic function $m_{0}(x) \in L_{2}([0,2 \pi])$ with period $2 \pi$ and such that $\widehat{\phi}(0) \neq 0$,

$$
\widehat{\phi}(y)=m_{0}[y / 2] \widehat{\phi}[y / 2],
$$

and $\widehat{\phi}(y)$ is continuous at the origin. In this case, the function $\phi(x)$ is called an $f$-wavelet.

Let $\psi(x)$ be the inverse Fourier transform of the function $\widehat{\psi}(y)$ satisfying the following functional equality:

$$
\widehat{\psi}(y)=\overline{m_{0}\left(\frac{y}{2}+\pi\right)} \cdot \exp \left\{-i \frac{y}{2}\right\} \cdot \widehat{\psi}\left(\frac{y}{2}\right)
$$

Then the function

$$
\psi(x)=\frac{1}{2 \pi} \int_{\mathbb{R}} e^{i y x} \widehat{\psi}(y) d y
$$

is called an $m$-wavelet.

Let

$$
\phi_{j k}(x)=2^{j / 2} \phi\left(2^{j} x-k\right), \quad \psi_{j k}(x)=2^{j / 2} \psi\left(2^{j} x-k\right), \quad j, k \in \mathbb{Z} .
$$

It is known that the family of functions $\left\{\phi_{0 k} ; \psi_{j k}, j=0,1,2, \ldots ; k \in \mathbb{Z}\right\}$ is an orthonormal basis in the space $L_{2}(\mathbb{R})$ (see, for example, [3, 4]).

Any function $f(x) \in L_{2}(\mathbb{R})$ can be represented in the form

$$
\begin{gathered}
f(x)=\sum_{k \in \mathbb{Z}} \alpha_{0 k} \phi_{0 k}(t)+\sum_{j=0}^{\infty} \sum_{k \in \mathbb{Z}} \beta_{j k} \psi_{j k}(t), \\
\alpha_{0 k}=\int_{\mathbb{R}} f(x) \overline{\phi_{0 k}(x)} d x, \quad \beta_{j k}=\int_{\mathbb{R}} f(x) \overline{\psi_{j k}(x)} d x .
\end{gathered}
$$

Representation (7) is called the wavelet decomposition.

The series (77) converge in the norm of the space $L_{2}(\mathbb{R})$, that is,

$$
\sum_{k \in \mathbb{Z}}\left|\alpha_{0 k}\right|^{2}+\sum_{j=0}^{\infty} \sum_{k \in \mathbb{Z}}\left|\beta_{j k}\right|^{2}<\infty
$$

The integrals determining $\alpha_{0 k}$ and $\beta_{j k}$ are well defined for functions in $L_{1}(\mathbb{R})$ or for those in some other spaces. Thus representation (7) may, in fact, hold for a larger class of functions than the space $L_{2}(\mathbb{R})$.

Let $\{\Omega, \mathfrak{F}, \mathrm{P}\}$ be a standard probability space. Let $X=\{X(t), t \in \mathbb{R}\}$ be a stochastic process and let $\mathrm{E} X(t)=0$.

Representation (7) can be proved for stochastic processes whose trajectories belong to the space $L_{2}(\mathbb{R})$ with probability one. However, many stochastic processes do not 
possess this property. For example, the trajectories of stationary processes do not belong to $L_{2}(\mathbb{R})$.

We study a representation for $X(t)$ similar to (7), namely

$$
X(t)=\sum_{k \in \mathbb{Z}} \xi_{0 k} \phi_{0 k}(t)+\sum_{j=0}^{\infty} \sum_{k \in \mathbb{Z}} \eta_{j k} \psi_{j k}(t),
$$

where the integrals

$$
\xi_{0 k}=\int_{\mathbb{R}} X(t) \overline{\phi_{0 k}(t)} d t, \quad \eta_{j k}=\int_{\mathbb{R}} X(t) \overline{\psi_{j k}(t)} d t
$$

are understood in the mean square sense.

We prove the uniform convergence of the wavelet decomposition (9) for the following approximation:

$$
X_{n, k_{j}}(t)=\sum_{|k| \leq k_{0}} \xi_{0 k} \phi_{0 k}(t)+\sum_{j=0}^{n-1} \sum_{|k| \leq k_{j}} \eta_{j k} \psi_{j k}(t) .
$$

Below we state Theorem 2.1 containing some sufficient conditions for the convergence in the norm of the space $L_{2}(\Omega)$ of wavelet decompositions. The proof of this result can be found in [7].

Definition $2.1([6])$. Let $\phi$ be an $f$-wavelet. We say that $\phi$ satisfies condition $S$ if there exists a function $\Phi=\{\Phi(x), x \geq 0\}$ such that $\Phi(0)<\infty, \Phi(x)$ decreases for $x \geq 0$, $|\phi(x)| \leq \Phi(|x|)$ almost everywhere, and

$$
\int_{\mathbb{R}} \Phi(|x|) d x<\infty
$$

Theorem 2.1. Let $X=\{X(t), t \in \mathbb{R}\}$ be a stochastic process such that $\mathrm{E} X(t)=0$, $\mathrm{E}|X(t)|^{2}<\infty$ for all $t \in \mathbb{R}$, and the covariance function $R_{X}(t, s)=R(t, s)$ is continuous. Let wavelets $\phi$ and $\psi$ be continuous functions and let condition $S$ hold for both of them.

Assume that $c=\{c(x), x \in \mathbb{R}\}$ is an even function such that $c(x)>1$ for all $x \in \mathbb{R}$. Assume further that the function $c$ is nondecreasing in the domain $x>0$ and there exists a function $0<A(a)<\infty$ defined for $a>0$ and such that

$$
c(a x) \leq c(x) \cdot A(a)
$$

for sufficiently large $x$.

If

$$
\int_{\mathbb{R}} c(x) \Phi(|x|) d x<\infty
$$

and $|R(t, t)|^{1 / 2} \leq c(t)$, then

1) $X_{n, k_{j}}(t) \in L_{2}(\Omega)$;

2) $X_{n, k_{j}}(t) \rightarrow X(t)$ as $n \rightarrow \infty$ and $k_{j} \rightarrow \infty$ for all $j=0,1, \ldots$ in the mean square sense, that is,

$$
\mathrm{E}\left|X_{n, k_{j}}(t)-X(t)\right|^{2} \rightarrow 0
$$

\section{Exponential type stochastic PROCEsses From ORLICZ SPACES}

Definition 3.1 ([1). A continuous even convex function $U=\{U(x), x \in \mathbb{R}\}$ is called a $C$-function if $U(0)=0$ and $U(x)$ increases for $x>0$.

Let $\{\Omega, \mathfrak{F}, \mathrm{P}\}$ be a standard probability space. 
Definition $3.2([1])$. Let $U(x)$ be an arbitrary $C$-function. A family of random variables $L_{U}(\Omega)$ is called an Orlicz space if, for every $\xi \in L_{U}(\Omega)$, there exists a constant $r_{\xi}>0$ such that $\mathrm{E} U\left(\xi / r_{\xi}\right)<\infty$.

Theorem 3.1 ([1]). An Orlicz space $L_{U}(\Omega)$ is a Banach space with respect to the Luxemburg norm

$$
\|\xi\|_{U}=\inf \{r>0: \mathrm{E} U(\xi / r) \leq 1\} .
$$

Definition 3.3 ([1]). The space $L_{U}(\Omega)$ is called an Orlicz space of exponential type if it is generated by a function

$$
U(x)=\exp \{\varphi(x)\}-1,
$$

where $\varphi(x)$ is a $C$-function. Following [1], Orlicz spaces of exponential type are denoted by $\operatorname{Exp}_{\varphi}(\Omega)$, while the norms in these space are denoted by $\|\cdot\|_{\operatorname{Exp}_{\varphi}}$.

Definition 3.4 ([1]). Let $\mathbb{T}$ be a set of parameters. We say that a stochastic process

$$
X=\{X(t), t \in \mathbb{T}\}
$$

belongs to a space $L_{U}(\Omega)$ if, for all $t \in \mathbb{T}$, the random variable $X(t)$ belongs to the space $L_{U}(\Omega)$.

Definition $3.5([14])$. We say that $X(t) \in L_{U}(\Omega)$ is a strictly Orlicz stochastic process if there is a constant $C_{T}$ such that, for all $t_{1}, t_{2}, \ldots, t_{n} \in \mathbb{T}$ and for all $c_{1}, c_{2}, \ldots, c_{n} \in \mathbb{R}$,

$$
\left\|\sum_{k=1}^{n} c_{k} X\left(t_{k}\right)\right\|_{U}<C_{T} \sqrt{\mathrm{E} \sum_{k=1}^{n} c_{k} X\left(t_{k}\right)} .
$$

Theorem 3.2. Let $\mathbb{T}=[0, T]$ and let

$$
X_{n}=\left\{X_{n}(t), t \in \mathbb{T}\right\}
$$

be a sequence of separable stochastic processes from an Orlicz space $L_{U}(\Omega)$. Assume that

1) there exists an increasing function $\sigma$ such that $\sigma(h) \rightarrow 0$ as $h \rightarrow 0$ and

$$
\sup _{n \geq 1} \rho(t, s)=\sup _{\substack{|t-s| \leq h \\ t, s \in \mathbb{T}}}\left\|X_{n}(t)-X_{n}(s)\right\|_{\operatorname{Exp}_{\varphi} \leq \sigma(h) ;}
$$

2) the processes $X_{n}(t)$ converge in probability as $n \rightarrow \infty$ to a separable process $X(t)$ for all points $t$

3) for all $\varepsilon>0$, the integral

$$
\int_{0}^{\varepsilon} U^{(-1)}\left(\frac{1}{2 \sigma^{(-1)}(t)}+1\right) d t<\infty
$$

converges.

Then the processes $X_{n}(t)$ and $X(t)$ are continuous and $X_{n}(t) \rightarrow X(t)$ as $n \rightarrow \infty$ uniformly in probability on $\mathbb{T}$.

This result follows from the bound

$$
N_{\rho}(t) \leq \frac{1}{2 \sigma^{(-1)}(t)}+1
$$

(see Theorem 3.2 in [10]). 
Lemma 3.1. Let the function $\sigma(u)$ in (11) be given by

$$
\sigma(u)=\frac{c}{\left(\ln \left(e^{\alpha}+1 / u\right)\right)^{\alpha}}, \quad 0<\alpha<1,
$$

and let $U(x)=\exp \{\varphi(x)\}-1$. Then condition (12) holds if

$$
\int_{0}^{\varepsilon} \varphi^{(-1)}\left(\left(\frac{c}{t}\right)^{1 / \alpha}\right) d t<\infty \text {. }
$$

Proof. Indeed,

and

$$
\sigma^{(-1)}(t)=\frac{1}{e^{(c / t)^{1 / \alpha}}-e^{\alpha}}
$$

$$
U^{-1}(t)=\varphi^{-1}(\ln (t+1)) .
$$

Then the integral in (12) is estimated as follows:

$$
\begin{aligned}
\int_{0}^{\varepsilon} U^{(-1)}\left(\frac{1}{2 \sigma^{(-1)}(t)}+1\right) d t & =\int_{0}^{\varepsilon} \varphi^{(-1)}\left(\ln \left(\frac{1}{2 \sigma^{(-1)}(t)}+2\right)\right) d t \\
& =\int_{0}^{\varepsilon} \varphi^{(-1)}\left(\ln \left(\frac{1}{2}\left(e^{(c / t)^{1 / \alpha}}-e^{\alpha}\right)+2\right)\right) d t \\
& \leq \int_{0}^{\varepsilon} \varphi^{(-1)}\left(\left(\frac{c}{t}\right)^{1 / \alpha}\right) d t<\infty
\end{aligned}
$$

4. UNIFORM CONVERGENCE OF WAVELET DECOMPOSITIONS FOR STOCHASTIC PROCESSES BELONGING TO ORLICZ SPACES OF EXPONENTIAL TYPE

Theorem 4.1. Let $X=\{X(t), t \in \mathbb{R}\}$ be a separable strictly Orlicz stochastic process in the space $\operatorname{Exp}_{\varphi}(\Omega)$ whose covariance function $R(t, s)$ is continuous. Suppose the assumptions of Theorem 2.1 as well as condition (13) hold. We further assume that, for an $f$-wavelet $\phi$ and for the corresponding $m$-wavelet $\psi$, the following conditions hold:

1) the derivatives $\widehat{\psi}^{\prime}(u)$ and $\widehat{\psi}^{\prime \prime}(u)$ exist for all $u$ and $\widehat{\psi}(0)=0$ and $\widehat{\psi}^{\prime}(0)=0$;

2) the constants $c_{\phi}, c_{\phi^{\prime}}$, and $c_{\psi^{\prime \prime}}$ are finite, where

$$
c_{\phi}=\sup _{u}|\widehat{\phi}(u)|, \quad c_{\phi^{\prime}}=\sup _{u}\left|\widehat{\phi}^{\prime}\right|, \quad \text { and } \quad c_{\psi^{\prime \prime}}=\sup _{u}\left|\widehat{\psi}^{\prime \prime}(u)\right| ;
$$

3) $\widehat{\phi}(u) \rightarrow 0$ as $u \rightarrow \pm \infty$ and $\widehat{\psi}(u) \rightarrow 0$ as $u \rightarrow \pm \infty$;

4) the following two integrals are well defined:

$$
\begin{aligned}
& \int_{\mathbb{R}}(\ln (1+|u|))^{\alpha}|\widehat{\psi}(u)|^{\beta} d u<\infty, \\
& \int_{\mathbb{R}}(\ln (1+|u|))^{\alpha}|\widehat{\phi}(u)|^{\beta} d u<\infty,
\end{aligned}
$$

where $0<\beta \leq 1$ and $0 \leq \alpha \leq 1$;

5) the following integrals exist:

$$
\int_{\mathbb{R}} \int_{\mathbb{R}}\left|\frac{\partial^{k+l} \widehat{R_{2}}(z, w)}{\partial w \partial z}\right||w|^{t}|z|^{s} d w d z<\infty, \quad k, l=0,1, \quad t, s=0,1,2 ;
$$

6) $\left|\widehat{R}_{2}(t, s)\right|<c<\infty$.

Then, for all $j=0,1, \ldots$, as $n \rightarrow \infty$ and $k_{j} \rightarrow \infty$,

$$
X_{n, k_{j}}(t) \rightarrow X(t)
$$

uniformly in probability in every interval $[0, T]$. 
Proof. Assumption 2) of Theorem 3.2 follows from Theorem 2.1. In view of Lemma 3.1. assumption (13) implies condition 3) of Theorem 3.2. Now we check that condition (11) holds for the representation $X_{n, k_{j}}(t)$ and the function

$$
\sigma(h)=\frac{B}{\left(\ln \left(e^{\alpha}+1 /|h|\right)\right)^{\alpha}}, \quad 0<\alpha<1 .
$$

Since $X(t)$ is a strictly Orlicz stochastic process, $X_{n, k_{j}}(t)$ is also a strictly Orlicz process (see [14]). Thus we need to check the inequality

$$
\begin{aligned}
\left\|X_{n, k_{j}}(t)-X_{n, k_{j}}(s)\right\|_{\operatorname{Exp}_{\varphi}} & \leq C_{T}\left(\mathrm{E}\left|X_{n, k_{j}}(t)-X_{n, k_{j}}(s)\right|^{2}\right)^{1 / 2} \\
& \leq \frac{\widetilde{B}}{\left(\ln \left(e^{\alpha}+1 /|t-s|\right)\right)^{\alpha}},
\end{aligned}
$$

where $\widetilde{B}$ is a certain constant. We have

$$
\begin{aligned}
& X_{n, k_{j}}(t)-X_{n, k_{j}}(s)=\sum_{|k| \leq k_{0}} \xi_{0 k}\left(\phi_{0 k}(t)-\phi_{0 k}(s)\right)+\sum_{j=0}^{n-1} \sum_{|k| \leq k_{j}} \eta_{j k}\left(\psi_{j k}(t)-\psi_{j k}(s)\right), \\
&\left(\mathrm{E}\left|X_{n, k_{j}}(t)-X_{n, k_{j}}(s)\right|^{2}\right)^{1 / 2} \leq\left(\mathrm{E}\left|\sum_{|k| \leq k_{0}} \xi_{0 k}\left(\phi_{0 k}(t)-\phi_{0 k}(s)\right)\right|^{2}\right)^{1 / 2} \\
&+\sum_{j=0}^{n-1}\left(\mathrm{E}\left|\sum_{|k| \leq k_{j}} \eta_{j k}\left(\psi_{j k}(t)-\psi_{j k}(s)\right)\right|^{2}\right)^{1 / 2} \\
&=\left(S_{1}\right)^{1 / 2}+\sum_{j=0}^{n-1}\left(S_{2}\right)^{1 / 2}
\end{aligned}
$$

Now we estimate $S_{2}$. A similar method applies to $S_{1}$. Indeed,

$$
S_{2} \leq \sum_{|k| \leq k_{j}} \sum_{|l| \leq k_{j}}\left|\mathrm{E} \eta_{j k} \eta_{j l}\right| \cdot\left|\psi_{j k}(t)-\psi_{j k}(s)\right| \cdot\left|\psi_{j l}(t)-\psi_{j l}(s)\right| .
$$

First we consider $\mathrm{E} \eta_{j k} \eta_{j l}$. Applying the Parseval equality, we get

$$
\begin{aligned}
\mathrm{E} \eta_{j k} \eta_{j l} & =\int_{\mathbb{R}} \int_{\mathbb{R}} \mathrm{E} \xi(u) \xi(v) \psi_{j k}(u) \psi_{j l}(v) d u d v \\
& =\frac{1}{(2 \pi)^{2}} \int_{\mathbb{R}} \int_{\mathbb{R}} \widehat{R_{2}}(z, w) \widehat{\psi}_{j k}(z) \widehat{\psi}_{j l}(w) d z d w,
\end{aligned}
$$

where

Note that

$$
\widehat{R_{2}}(z, w)=\int_{\mathbb{R}} \int_{\mathbb{R}} R(u, v) e^{-i z u} e^{-i w v} d u d v
$$

$$
\widehat{\psi}_{j k}(z)=\frac{\exp \left\{-i\left(k / 2^{j}\right) z\right\}}{2^{j / 2}} \cdot \widehat{\psi}\left(\frac{z}{2^{j}}\right) .
$$

In the next transformation we use integration by parts, assumptions 1), 2), and 5) of the theorem, and the inequality

$$
\left|\widehat{\psi}\left(\frac{z}{2^{j}}\right)\right|=\left|\frac{\widehat{\psi}^{\prime \prime}(\tilde{z})}{2}\left(\frac{z}{2^{j}}\right)^{2}\right| \leq c_{\psi^{\prime \prime}} \frac{|z|^{2}}{2^{2 j}} .
$$


Thus

$$
\begin{aligned}
&\left|\mathrm{E} \eta_{j k} \eta_{j l}\right|=\mid \frac{1}{(2 \pi)^{2}} \int_{\mathbb{R}} \int_{\mathbb{R}} \widehat{R_{2}}(z, w) \frac{\exp \left\{-i\left(k / 2^{j}\right) w\right\}}{2^{j / 2}} \widehat{\psi}\left(\frac{w}{2^{j}}\right) \\
& \times \frac{\exp \left\{-i\left(l / 2^{j}\right) z\right\}}{2^{j / 2}} \widehat{\psi}\left(\frac{z}{2^{j}}\right) d w d z \mid \\
&=\frac{2^{j}}{(2 \pi)^{2}|k||l|} \mid \int_{\mathbb{R}} \int_{\mathbb{R}}\left(\frac{\partial^{2} \widehat{R_{2}}(z, w)}{\partial w \partial z} \widehat{\psi}\left(\frac{z}{2^{j}}\right) \widehat{\psi}\left(\frac{w}{2^{j}}\right)\right.+\frac{\partial \widehat{R_{2}}(z, w)}{\partial z} \frac{1}{2^{j}} \widehat{\psi}^{\prime}\left(\frac{w}{2^{j}}\right) \widehat{\psi}\left(\frac{z}{2^{j}}\right) \\
&+\frac{\partial \widehat{R_{2}}(z, w)}{\partial w} \widehat{\psi}\left(\frac{w}{2^{j}}\right) \frac{1}{2^{j}} \widehat{\psi}^{\prime}\left(\frac{z}{2^{j}}\right) \\
&\left.+\frac{\widehat{R_{2}}(z, w)}{2^{j}} \widehat{\psi^{\prime}}\left(\frac{w}{2^{j}}\right) \frac{1}{2^{j}} \widehat{\psi}^{\prime}\left(\frac{z}{2^{j}}\right)\right) \\
& \times \exp \left\{-i \frac{k}{2^{j}} w\right\} d w \exp \left\{-i \frac{l}{2^{j}} z\right\} d z \mid \\
& \leq \frac{1}{|k||l| 2^{3 j}} A^{\psi}, \quad \\
&
\end{aligned}
$$

where

$$
\begin{aligned}
A^{\psi}= & \frac{c_{\psi^{\prime \prime}}^{2}}{(2 \pi)^{2}} \int_{\mathbb{R}} \int_{\mathbb{R}}\left(\left|\frac{\partial^{2} \widehat{R_{2}}(z, w)}{\partial w \partial z}\right||w|^{2}|z|^{2}+\left|\frac{\partial \widehat{R_{2}}(z, w)}{\partial z}\right||w| \cdot|z|^{2}\right. \\
& \left.+\left|\frac{\partial \widehat{R_{2}}(z, w)}{\partial w}\right||w|^{2}|z|+\left|\widehat{R_{2}}(z, w)\right| \cdot|w| \cdot|z|\right) d w d z \\
& <\infty .
\end{aligned}
$$

To estimate the difference $\left|\psi_{j l}(t)-\psi_{j l}(s)\right|$, we use equality (14). Then

$$
\begin{gathered}
\psi_{j l}(t)=\frac{1}{(2 \pi)} \int_{\mathbb{R}} e^{i t z} \frac{1}{2^{j / 2}} \exp \left\{-i \frac{l}{2^{j}} z\right\} \widehat{\psi}\left(\frac{z}{2^{j}}\right) d z \\
\psi_{j l}(t)=\frac{1}{(2 \pi) 2^{j / 2}} \int_{\mathbb{R}} \exp \left\{i t\left(z+\frac{2^{j}}{l} \pi\right)\right\} \exp \left\{-i \frac{l}{2^{j}} z\right\} e^{-i \pi} \widehat{\psi}\left(\frac{z}{2^{j}}+\frac{\pi}{l}\right) d z .
\end{gathered}
$$

Hence

$$
\psi_{j l}(t)=\frac{1}{(4 \pi) 2^{j / 2}} \int_{\mathbb{R}} \exp \left\{-i \frac{l}{2^{j}} z\right\}\left(e^{i t z} \widehat{\psi}\left(\frac{z}{2^{j}}\right)-\exp \left\{i t\left(z+\frac{2^{j}}{l} \pi\right)\right\} \widehat{\psi}\left(\frac{z}{2^{j}}+\frac{\pi}{l}\right)\right) d z
$$


whence

$$
\begin{aligned}
& \left|\psi_{j l}(t)-\psi_{j l}(s)\right| \\
& =\frac{1}{(4 \pi)} \frac{1}{2^{j / 2}} \mid \int_{\mathbb{R}} \exp \left\{-i \frac{l}{2^{j}} z\right\} \\
& \times\left[\left(e^{i t z} \widehat{\psi}\left(\frac{z}{2^{j}}\right)-\exp \left\{i t\left(z+\frac{2^{j}}{l} \pi\right)\right\} \widehat{\psi}\left(\frac{z}{2^{j}}+\frac{\pi}{l}\right)\right)\right. \\
& \left.-\left(e^{i s z} \widehat{\psi}\left(\frac{z}{2^{j}}\right)-\exp \left\{i s\left(z+\frac{2^{j}}{l} \pi\right)\right\} \widehat{\psi}\left(\frac{z}{2^{j}}+\frac{\pi}{l}\right)\right)\right] d z \mid \\
& \leq \frac{1}{(4 \pi)} \frac{1}{2^{j / 2}} \int_{\mathbb{R}}\left(\left|e^{i t z}-e^{i s z}-\exp \left\{i t\left(z+\frac{2^{j}}{l} \pi\right)\right\}+\exp \left\{i s\left(z+\frac{2^{j}}{l} \pi\right)\right\}\right|\right. \\
& \times\left|\widehat{\psi}\left(\frac{z}{2^{j}}\right)\right| \\
& +\left|\exp \left\{i t\left(z+\frac{2^{j}}{l} \pi\right)\right\}-\exp \left\{i s\left(z+\frac{2^{j}}{l} \pi\right)\right\}\right| \\
& \left.\times\left|\widehat{\psi}\left(\frac{z}{2^{j}}\right)-\widehat{\psi}\left(\frac{z}{2^{j}}+\frac{\pi}{l}\right)\right|\right) d z \\
& =\left|\frac{z}{2^{j}}=u\right| \\
& =\frac{2^{j / 2}}{(4 \pi)} \int_{\mathbb{R}}\left(\mid \exp \left\{i t 2^{j} u\right\}-\exp \left\{i s 2^{j} u\right\}-\exp \left\{i t 2^{j}\left(u+\frac{\pi}{l}\right)\right\}\right. \\
& +\exp \left\{i s 2^{j}\left(u+\frac{\pi}{l}\right)\right\}|| \widehat{\psi}(u) \mid \\
& +\left|\exp \left\{i t 2^{j}\left(u+\frac{\pi}{l}\right)\right\}-\exp \left\{i s 2^{j}\left(u+\frac{\pi}{l}\right)\right\}\right| \\
& \left.\times\left|\widehat{\psi}(u)-\widehat{\psi}\left(u+\frac{\pi}{l}\right)\right|\right) d u .
\end{aligned}
$$

To estimate the latter integral, we use the inequality

$$
\left|e^{i t z}-e^{i s z}\right| \leq 2\left|\sin \frac{z(t-s)}{2}\right| \leq 2\left(\frac{\ln \left(e^{\alpha}+z / 2\right)}{\ln \left(e^{\alpha}+1 /|t-s|\right)}\right)^{\alpha},
$$

(see, for example, [13]). We estimate the second term of the latter integral as follows:

$$
\begin{aligned}
I_{2} & =\int_{\mathbb{R}}\left|\exp \left\{i t 2^{j}\left(u+\frac{\pi}{l}\right)\right\}-\exp \left\{i s 2^{j}\left(u+\frac{\pi}{l}\right)\right\}\right|\left|\widehat{\psi}(u)-\widehat{\psi}\left(u+\frac{\pi}{l}\right)\right| d u \\
& =\left|u+\frac{\pi}{l}=v\right| \\
& =\int_{\mathbb{R}}\left|\exp \left\{i t 2^{j} v\right\}-\exp \left\{i s 2^{j} v\right\}\right|\left|\widehat{\psi}\left(v-\frac{\pi}{l}\right)-\widehat{\psi}(v)\right| d v \\
& \leq \frac{2}{\left(\ln \left(e^{\alpha}+\frac{1}{|t-s|}\right)\right)^{\alpha}} \int_{\mathbb{R}}\left(\ln \left(e^{\alpha}+\frac{|v| 2^{j}}{2}\right)\right)^{\alpha}\left|\widehat{\psi}\left(v-\frac{\pi}{l}\right)-\widehat{\psi}(v)\right|^{\beta}\left|\widehat{\psi}\left(v-\frac{\pi}{l}\right)-\widehat{\psi}(v)\right|^{1-\beta} d v
\end{aligned}
$$


In the next transformation, we apply assumptions 1) and 4) of Theorem 4.1 and the inequalities

$$
\begin{gathered}
\left|\widehat{\psi}\left(v-\frac{\pi}{l}\right)-\widehat{\psi}(v)\right|^{\beta}=\frac{b}{|l|^{\beta}}, \quad b=a \pi^{\beta} \\
\left|\widehat{\psi}\left(v-\frac{\pi}{l}\right)-\widehat{\psi}(v)\right|^{1-\beta} \leq\left(\left|\widehat{\psi}\left(v-\frac{\pi}{l}\right)\right|^{1-\beta}+|\widehat{\psi}(v)|^{1-\beta}\right),
\end{gathered}
$$

and

$$
\begin{aligned}
& \int_{\mathbb{R}} b\left(\ln \left(e^{\alpha}+\frac{|v| 2^{j}}{2}\right)\right)^{\alpha}\left|\widehat{\psi}\left(v-\frac{\pi}{l}\right)\right|^{1-\beta} d v \\
& \leq b \int_{\mathbb{R}}\left(\ln \left(2 e^{\alpha}+|v|+\pi\right)^{j}\right)^{\alpha}|\widehat{\psi}(v)|^{1-\beta} d v=j^{\alpha} c_{1}<\infty \\
& c_{1}=b \int_{\mathbb{R}}\left(\ln \left(2 e^{\alpha}+\pi+|v|\right)\right)^{\alpha}|\widehat{\psi}(v)|^{1-\beta} d v<\infty
\end{aligned}
$$

From these results we have

$$
I_{2} \leq \frac{2}{\left(\ln \left(e^{\alpha}+1 /|t-s|\right)\right)^{\alpha}} \frac{1}{|l|^{\beta}} j^{\alpha} 2 c_{1} .
$$

The first term of the same integral (we call it $I_{1}$ ) can be estimated similarly. First we use the inequality

$$
\begin{aligned}
& \left|\exp \left\{i t 2^{j} u\right\}-\exp \left\{i s 2^{j} u\right\}-\exp \left\{i t 2^{j}\left(u+\frac{\pi}{l}\right)\right\}+\exp \left\{i s 2^{j}\left(u+\frac{\pi}{l}\right)\right\}\right| \\
& \quad \leq\left|\exp \left\{i t 2^{j} u\right\}-\exp \left\{i s 2^{j} u\right\}\right|\left|1-\exp \left\{i t 2^{j} \frac{\pi}{l}\right\}\right|+\left|\exp \left\{i s 2^{j} \frac{\pi}{l}\right\}-\exp \left\{i t 2^{j} \frac{\pi}{l}\right\}\right| \\
& \quad=:|\Delta| \\
& \quad \leq 2\left|\sin \frac{2^{j} u(t-s)}{2}\right| \cdot\left|\sin \frac{\pi}{l} 2^{j} \frac{t}{2}\right|+2\left|\sin \frac{(t-s)}{2} \frac{\pi}{l} 2^{j}\right| .
\end{aligned}
$$

Note that

$$
\begin{aligned}
&\left|\sin \frac{2^{j} u(t-s)}{2}\right| \leq\left(\frac{\ln \left(e^{\alpha}+\frac{2^{j}|u|}{2}\right)}{\ln \left(e^{\alpha}+1 /|t-s|\right)}\right)^{\alpha}, \quad 0<\alpha \leq 1, \\
&\left|\sin \frac{\pi}{l} 2^{j} \frac{t}{2}\right| \leq\left(\pi \frac{t}{2}\right)^{\beta} \cdot\left(\frac{2^{j}}{|l|}\right)^{\beta} \leq\left(\pi \frac{T}{2}\right)^{\beta} \cdot \frac{2^{j \beta}}{|l|^{\beta}}, \\
&\left|\sin \frac{(t-s)}{2} \frac{\pi}{l} 2^{j}\right| \leq\left(\frac{\pi 2^{j}}{|l|}\right)^{\beta} \cdot\left(\frac{|s-t|}{2}\right)^{\beta} \leq\left(\frac{2^{j}}{|l|}\right)^{\beta} \frac{c_{\alpha, \beta, T}}{\left(\ln \left(e^{\alpha}+1 /|t-s|\right)\right)^{\alpha}} .
\end{aligned}
$$

Then

$$
|\Delta| \leq \frac{2^{j \beta}}{|l|^{\beta}} \cdot \frac{j^{\alpha}}{\left(\ln \left(e^{\alpha}+1 /|t-s|\right)\right)^{\alpha}}\left(2\left(\pi \frac{T}{2}\right)^{\beta}(\ln (1+|u|))^{\alpha}+2 c_{\alpha, \beta, T}\right) .
$$

Now the first part of the integral is estimated as follows:

$$
I_{1} \leq \frac{2^{j \beta}}{l^{\beta}} \cdot \frac{j^{\alpha}}{\left(\ln \left(e^{\alpha}+1 /|t-s|\right)\right)^{\alpha}} \cdot c_{2}
$$

where

$$
c_{2}=\int_{\mathbb{R}}\left(2\left(\pi \frac{T}{2}\right)^{\beta}(\ln (1+|u|))^{\alpha}+2 c_{\alpha, \beta, T}\right)|\widehat{\psi}(u)| d u<\infty .
$$


Taking into account the bounds (18) and (19), we obtain

$$
\begin{aligned}
\mid \psi_{j k}(t) & -\psi_{j k}(s)|\cdot| \psi_{j l}(t)-\psi_{j l}(s) \mid \\
\leq & \frac{2^{j}}{(4 \pi)^{2}} \frac{j^{2 \alpha}}{\left(\ln \left(e^{\alpha}+1 /|t-s|\right)\right)^{2 \alpha}}\left(\frac{2^{j \beta}}{|l|^{\beta}} \cdot c_{2}+\frac{4 c_{1}}{|l|^{\beta}}\right)\left(\frac{2^{j \beta}}{|k|^{\beta}} \cdot c_{2}+\frac{4 c_{1}}{|k|^{\beta}}\right) \\
\leq & \frac{2^{j(1+2 \beta)} j^{2 \alpha}}{\left(\ln \left(e^{\alpha}+1 /|t-s|\right)\right)^{2 \alpha}} \frac{1}{|l|^{\beta}} \frac{1}{|k|^{\beta}} \cdot\left(c^{\psi}\right)^{2}
\end{aligned}
$$

where $c^{\psi}=\left(c_{2}+4 c_{1}\right) /(4 \pi)$. Therefore, for the case where $l \neq 0$ and $k \neq 0$, we have that

$$
\begin{aligned}
& \sum_{\substack{|k| \leq k_{j}, k \neq 0}} \sum_{\substack{|l| \leq k_{j}, l \neq 0}}\left|\mathrm{E} \eta_{j k} \eta_{j l}\right|\left|\psi_{j k}(t)-\psi_{j k}(s)\right|\left|\psi_{j l}(t)-\psi_{j l}(s)\right| \\
& \quad \leq \sum_{\substack{|k| \leq k_{j},|l| \leq k_{j}, k \neq 0}} \frac{1}{2^{3 j}} \frac{1}{|k||l|} \cdot A^{\psi} \frac{2^{(2 \beta+1) j}}{|l|^{\beta}|k|^{\beta}}\left(c^{\psi}\right)^{2} \frac{j^{2 \alpha}}{\left(\ln \left(e^{\alpha}+1 /|t-s|\right)\right)^{2 \alpha}} \\
& \quad=\frac{j^{2 \alpha}}{2^{(2-2 \beta) j}} \frac{A^{\psi}\left(c^{\psi}\right)^{2} \hat{q}^{2}}{\left(\ln \left(e^{\alpha}+1 /|t-s|\right)\right)^{2 \alpha}},
\end{aligned}
$$

where

$$
\hat{q}^{2}=\sum_{\substack{|k| \leq k_{j},|l| \leq k_{j}, k \neq 0}} \frac{1}{|l||k|} \cdot \frac{1}{|l|^{\beta}|k|^{\beta}} \leq \sum_{0<k \leq k_{j}} \sum_{0<l \leq k_{j}} \frac{2}{k^{1+\beta}} \cdot \frac{2}{l^{1+\beta}}<\infty .
$$

The series on the right hand side converges if $\beta>0$.

The case of $l \neq 0, k=0$ can be treated similarly. Thus

$$
\begin{aligned}
& \left|\mathrm{E} \eta_{j 0} \eta_{j l}\right|=\frac{1}{2 \pi}\left|\int_{\mathbb{R}} \widehat{R_{2}}(z, w) \frac{1}{2^{j / 2}} \widehat{\psi}\left(\frac{w}{2^{j}}\right) \cdot \frac{\exp \left\{-i \frac{l}{2^{j}} z\right\}}{2^{j / 2}} \widehat{\psi}\left(\frac{z}{2^{j}}\right) d z\right| \\
& =\frac{1}{(2 \pi)^{2}|l|} \mid \int_{\mathbb{R}} \int_{\mathbb{R}}\left(\frac{\partial \widehat{R}_{2}(z, w)}{\partial z} \widehat{\psi}\left(\frac{z}{2^{j}}\right)+\frac{\widehat{R}_{2}(z, w)}{2^{j}} \widehat{\psi}^{\prime}\left(\frac{z}{2^{j}}\right)\right) \\
& \times \exp \left\{-i \frac{l}{2^{j}} z\right\} \widehat{\psi}\left(\frac{w}{2^{j}}\right) d z d w \mid \\
& \leq \frac{1}{2^{4 j}} \cdot \frac{1}{|l|} \cdot A_{1}^{\psi}
\end{aligned}
$$

where

$$
A_{1}^{\psi}=\frac{\left(c_{\psi^{\prime \prime}}\right)^{2}}{(2 \pi)^{2}} \int_{\mathbb{R}} \int_{\mathbb{R}}\left(\left|\frac{\partial \widehat{R}_{2}(z, w)}{\partial z}\right||z|^{2}|w|^{2}+\left|\widehat{R_{2}}(z, w)\right||z||w|^{2}\right) d z d w<\infty .
$$

Similar reasoning is applied in the case of $k \neq 0, l=0$. Hence

$$
\left|\mathrm{E} \eta_{j k} \eta_{j 0}\right| \leq \frac{A_{1}^{\psi}}{2^{4 j}|k|}
$$


Note that

$$
\begin{aligned}
\left|\psi_{j 0}(t)-\psi_{j 0}(s)\right| & =\frac{1}{(2 \pi)} \frac{1}{2^{j / 2}}\left|\int_{\mathbb{R}}\left(e^{i t z}-e^{i s z}\right) \widehat{\psi}\left(\frac{z}{2^{j}}\right) d z\right| \\
& =\frac{2^{j / 2}}{(2 \pi)}\left|\int_{\mathbb{R}}\left(e^{i t 2^{j} u}-e^{i s 2^{j} u}\right) \widehat{\psi}(u) d u\right| \\
& \leq \frac{2^{j / 2}}{(2 \pi)} \int_{\mathbb{R}}\left(\frac{\ln \left(e^{\alpha}+\frac{2^{j}|u|}{2}\right)}{\ln \left(e^{\alpha}+1 /|t-s|\right)}\right)^{\alpha}|\widehat{\psi}(u)| d u \\
& \leq \frac{2^{j / 2}}{(2 \pi)} \frac{j^{\alpha}}{\left(\ln \left(e^{\alpha}+1 /|t-s|\right)\right)^{\alpha}} \int_{\mathbb{R}}\left(\ln \left(2 e^{\alpha}+|u|\right)\right)^{\alpha}|\widehat{\psi}(u)| d u \\
& =\frac{2^{j / 2} j^{\alpha} \cdot c_{1}^{\psi}}{\left(\ln \left(e^{\alpha}+1 /|t-s|\right)\right)^{\alpha}},
\end{aligned}
$$

where

$$
c_{1}^{\psi}=\frac{1}{2 \pi} \int_{\mathbb{R}}\left(\ln \left(2 e^{\alpha}+|u|\right)\right)^{\alpha}|\widehat{\psi}(u)| d u<\infty .
$$

Then

$$
\begin{aligned}
& \sum_{|l| \leq k_{j}, l \neq 0}\left|\mathrm{E} \eta_{j 0} \eta_{j l}\right|\left|\psi_{j 0}(t)-\psi_{j 0}(s)\right|\left|\psi_{j l}(t)-\psi_{j l}(s)\right| \\
& \quad \leq \sum_{|l| \leq k_{j}, l \neq 0} \frac{1}{2^{4 j}} \frac{1}{|l|} \cdot A_{1}^{\psi} \frac{2^{j(1 / 2+\beta)} j^{\alpha}}{\left(\ln \left(e^{\alpha}+1 /|t-s|\right)\right)^{\alpha}} \frac{1}{|l|^{\beta}} \cdot c^{\psi} \frac{2^{j / 2} j^{\alpha} \cdot c_{1}^{\psi}}{\left(\ln \left(e^{\alpha}+1 /|t-s|\right)\right)^{\alpha}} \\
& \quad \leq \frac{j^{2 \alpha}}{2^{(3-\beta) j}} \cdot \frac{A_{1}^{\psi} \cdot c^{\psi} \cdot c_{1}^{\psi} \cdot q}{\left(\ln \left(e^{\alpha}+1 /|t-s|\right)\right)^{2 \alpha}}
\end{aligned}
$$

where

$$
q=\sum_{|l| \leq k_{j}, l \neq 0} \frac{1}{|l|^{1+\beta}}<\infty
$$

and

$$
\begin{aligned}
& \sum_{|k| \leq k_{j}, k \neq 0}\left|\mathrm{E} \eta_{j 0} \eta_{j k}\right|\left|\psi_{j 0}(t)-\psi_{j 0}(s)\right|\left|\psi_{j k}(t)-\psi_{j k}(s)\right| \\
& \quad \leq \frac{j^{2 \alpha}}{2^{(3-\beta) j}} \cdot \frac{A_{1}^{\psi} \cdot c^{\psi} \cdot c_{1}^{\psi} \cdot q}{\left(\ln \left(e^{\alpha}+1 /|t-s|\right)\right)^{2 \alpha}} .
\end{aligned}
$$

Using the bounds (21), (23), and (24), we get

$$
\begin{aligned}
& \sum_{j=0}^{n-1}\left(\mathrm{E}\left|\sum_{|k| \leq k_{j}} \eta_{j k}\left(\psi_{j k}(t)-\psi_{j k}(s)\right)\right|^{2}\right)^{1 / 2} \\
& \quad \leq \sum_{j=0}^{n-1}\left(\frac{j^{2 \alpha}}{2^{(2-2 \beta) j}} \frac{A^{\psi}\left(c^{\psi}\right)^{2} \hat{q}^{2}}{\left(\ln \left(e^{\alpha}+1 /|t-s|\right)\right)^{2 \alpha}}+2 \frac{j^{2 \alpha}}{2^{(3-\beta) j}} \cdot \frac{A_{1}^{\psi} \cdot c^{\psi} \cdot c_{1}^{\psi} \cdot q}{\left(\ln \left(e^{\alpha}+1 /|t-s|\right)\right)^{2 \alpha}}\right)^{1 / 2} \\
& \quad \leq q_{1}\left[c^{\psi}\left(c^{\psi} A^{\psi} \hat{q}^{2}+2 c_{1}^{\psi} A_{1}^{\psi} q\right)\right]^{1 / 2} \frac{1}{\left(\ln \left(e^{\alpha}+1 /|t-s|\right)\right)^{\alpha}}
\end{aligned}
$$


where

$$
q_{1}=\sum_{j=0}^{n-1} \frac{j^{\alpha}}{2^{(1-\beta) j}}<\infty, \quad \beta<1 .
$$

An analogous method is applied to

$$
S_{1}=\left(\mathrm{E}\left|\sum_{|k| \leq k_{0}} \xi_{0 k}\left(\phi_{0 k}(t)-\phi_{0 k}(s)\right)\right|^{2}\right)^{1 / 2} .
$$

Namely, we estimate $\left|\mathrm{E} \xi_{0 k} \xi_{0 l}\right|$ in the case of $k \neq 0, l \neq 0$ :

$$
\begin{aligned}
& \left|\mathrm{E} \xi_{0 k} \xi_{0 l}\right|=\left|\frac{1}{(2 \pi)^{2}} \int_{\mathbb{R}} \int_{\mathbb{R}} \widehat{R}_{2}(z, w) e^{-i k w} \widehat{\phi}(z) e^{-i l z} \widehat{\phi}(w) d z d w\right| \\
& =\frac{1}{(2 \pi)^{2}} \frac{1}{|k|}\left|\int_{\mathbb{R}} \int_{\mathbb{R}}\left(\frac{\partial \widehat{R}_{2}(z, w)}{\partial w} \widehat{\phi}(w)+\widehat{R}_{2}(z, w) \widehat{\phi}^{\prime}(w)\right) e^{-i k w} d w e^{-i l z} \widehat{\phi}(z) d z\right| \\
& =\frac{1}{(2 \pi)^{2}} \frac{1}{|k||l|} \mid \int_{\mathbb{R}} \int_{\mathbb{R}}\left(\frac{\partial^{2} \widehat{R}_{2}(z, w)}{\partial w \partial z} \widehat{\phi}(w) \widehat{\phi}(z)+\frac{\partial \widehat{R}_{2}(z, w)}{\partial z} \widehat{\phi}^{\prime}(w) \widehat{\phi}(z)\right. \\
& \left.+\frac{\partial \widehat{R}_{2}(z, w)}{\partial w} \widehat{\phi}(w) \widehat{\phi}^{\prime}(z)+\widehat{R}_{2}(z, w) \widehat{\phi}^{\prime}(w) \widehat{\phi}^{\prime}(z)\right) \\
& \leq \frac{1}{|k| \cdot|l|} \cdot A^{\phi},
\end{aligned}
$$

where

$$
\begin{aligned}
A^{\phi}=\frac{1}{(2 \pi)^{2}}\left(c_{\phi}^{2} \int_{\mathbb{R}} \int_{\mathbb{R}}\left|\frac{\partial^{2} \widehat{R}_{2}(z, w)}{\partial w \partial z}\right| d z d w\right. \\
\left.+2 c_{\phi} c_{\phi}^{\prime} \int_{\mathbb{R}} \int_{\mathbb{R}}\left|\frac{\partial \widehat{R}_{2}(z, w)}{\partial z}\right| d z d w+\left(c_{\phi}^{\prime}\right)^{2} \int_{\mathbb{R}} \int_{\mathbb{R}}\left|\widehat{R}_{2}(z, w)\right| d z d w\right)<\infty, \\
c_{\phi}=\sup _{u}|\widehat{\phi}(u)|<\infty, \quad c_{\phi}^{\prime}=\sup _{u}\left|\widehat{\phi}^{\prime}(u)\right|<\infty .
\end{aligned}
$$

Similarly to (20) we derive the bound

$$
\left|\phi_{0 k}(t)-\phi_{0 k}(s)\right| \cdot\left|\phi_{0 l}(t)-\phi_{0 l}(s)\right| \leq \frac{\left(c^{\phi}\right)^{2}}{\left(\ln \left(e^{\alpha}+1 /|t-s|\right)\right)^{2 \alpha}} \frac{1}{|l|^{\beta}} \frac{1}{|k|^{\beta}},
$$

where

$$
\begin{aligned}
c^{\phi}=\frac{1}{(4 \pi)}\left(4 \int _ { \mathbb { R } } \left(2\left(\pi \frac{T}{2}\right)^{\beta}(\right.\right. & \left.\ln (1+|u|))^{\alpha}+2 c_{\alpha}\right)|\widehat{\phi}(u)| d u \\
& \left.+\int_{\mathbb{R}}(\ln (1+|u|))^{\alpha}|\widehat{\phi}(u)|^{1-\beta} d u\right)<\infty
\end{aligned}
$$


Thus

$$
\begin{aligned}
& \sum_{|k| \leq k_{0}, k \neq 0} \sum_{|l| \leq k_{0}, l \neq 0}\left|\mathrm{E} \xi_{0 k} \xi_{0 l}\right|\left|\phi_{0 k}(t)-\phi_{0 k}(s)\right|\left|\phi_{0 l}(t)-\phi_{0 l}(s)\right| \\
& \quad \leq \sum_{|k| \leq k_{0}, k \neq 0} \sum_{|l| \leq k_{0}, l \neq 0} \frac{1}{|k||l|} \cdot A^{\phi} \frac{\left(c^{\phi}\right)^{2}}{\left(\ln \left(e^{\alpha}+1 /|t-s|\right)\right)^{2 \alpha}} \frac{1}{|l|^{\beta}} \frac{1}{|k|^{\beta}} \\
& \quad \leq \frac{A^{\phi}\left(c^{\phi}\right)^{2} \hat{q}^{2}}{\left(\ln \left(e^{\alpha}+1 /|t-s|\right)\right)^{2 \alpha}} .
\end{aligned}
$$

Consider the case of $k=0, l \neq 0$ :

$$
\begin{aligned}
\left|\mathrm{E} \xi_{00} \xi_{0 l}\right| & =\left|\frac{1}{(2 \pi)^{2}} \int_{\mathbb{R}} \int_{\mathbb{R}} \widehat{R}_{2}(z, w) \widehat{\phi}(w) \cdot e^{-i l z} \widehat{\phi}(z) d z d w\right| \\
& =\frac{1}{(2 \pi)^{2}} \frac{1}{|l|}\left|\int_{\mathbb{R}} \int_{\mathbb{R}}\left(\frac{\partial \widehat{R}_{2}(z, w)}{\partial z} \widehat{\phi}(z)+\widehat{R}_{2}(z, w) \widehat{\phi}^{\prime}(z)\right) e^{-i l z} d z \widehat{\phi}(w) d w\right| \\
& \leq \frac{1}{|l|} \cdot A_{1}^{\phi}
\end{aligned}
$$

where

$$
A_{1}^{\phi}=\frac{c_{\phi}}{(2 \pi)^{2}}\left(c_{\phi} \int_{\mathbb{R}} \int_{\mathbb{R}}\left|\frac{\partial \widehat{R}_{2}(z, w)}{\partial z}\right| d z d w+c_{\phi}^{\prime} \int_{\mathbb{R}} \int_{\mathbb{R}}\left|\widehat{R}_{2}(z, w)\right| d z d w\right)<\infty .
$$

In the case of $l=0, k \neq 0$, we have $\left|\mathrm{E} \xi_{00} \xi_{0 k}\right| \leq A_{1}^{\phi} /|k|$.

From the inequality (17) we get

$$
\begin{aligned}
\left|\phi_{00}(t)-\phi_{00}(s)\right| & =\frac{1}{(2 \pi)}\left|\int_{\mathbb{R}}\left(e^{i t z}-e^{i s z}\right) \widehat{\phi}(z) d z\right| \\
& \leq \frac{1}{\pi} \int_{\mathbb{R}}\left(\frac{\ln \left(e^{\alpha}+|z| / 2\right)}{\ln \left(e^{\alpha}+1 /|t-s|\right)}\right)^{\alpha}|\widehat{\phi}(z)| d z \leq \frac{c_{1}^{\phi}}{\left(\ln \left(e^{\alpha}+1 /|t-s|\right)\right)^{\alpha}},
\end{aligned}
$$

where

$$
c_{1}^{\phi}=\pi^{-1} \int_{\mathbb{R}}\left(\ln \left(2 e^{\alpha}+|z|\right)\right)^{\alpha}|\widehat{\phi}(z)| d z<\infty,
$$

whence we deduce that

$$
\begin{aligned}
& \sum_{|l| \leq k_{0}, l \neq 0}\left|\mathrm{E} \xi_{00} \xi_{0 l}\right| \cdot\left|\phi_{00}(t)-\phi_{00}(s)\right| \cdot\left|\phi_{0 l}(t)-\phi_{0 l}(s)\right| \\
& \quad \leq \sum_{|l| \leq k_{0}, l \neq 0} \frac{1}{|l|} \cdot A_{1}^{\phi} \frac{c_{1}^{\phi}}{\left(\ln \left(e^{\alpha}+1 /|t-s|\right)\right)^{\alpha}} \frac{1}{|l|^{\beta}} \frac{c^{\phi}}{\left(\ln \left(e^{\alpha}+1 /|t-s|\right)\right)^{\alpha}} \\
& \quad=\frac{A_{1}^{\phi} \cdot c^{\phi} \cdot c_{1}^{\phi} \cdot q}{\left(\ln \left(e^{\alpha}+1 /|t-s|\right)\right)^{2 \alpha}}
\end{aligned}
$$

and

(30) $\sum_{|k| \leq k_{0}, k \neq 0}\left|\mathrm{E} \xi_{00} \xi_{0 l}\right| \cdot\left|\phi_{00}(t)-\phi_{00}(s)\right| \cdot\left|\phi_{0 k}(t)-\phi_{0 k}(s)\right| \leq \frac{A_{1}^{\phi} \cdot c^{\phi} \cdot c_{1}^{\phi} \cdot q}{\left(\ln \left(e^{\alpha}+1 /|t-s|\right)\right)^{2 \alpha}}$. 
Using the bounds (28), (29), and (30), we obtain

$$
\begin{aligned}
(\mathrm{E} \mid & \left.\left.\sum_{|k| \leq k_{0}} \xi_{0 k}\left(\phi_{0 k}(t)-\phi_{0 k}(s)\right)\right|^{2}\right)^{1 / 2} \\
& \leq\left(\frac{A^{\phi}\left(c^{\phi}\right)^{2} \hat{q}^{2}}{\left(\ln \left(e^{\alpha}+1 /|t-s|\right)\right)^{2 \alpha}}+2 \frac{A_{1}^{\phi} \cdot c^{\phi} \cdot c_{1}^{\phi} \cdot q}{\left(\ln \left(e^{\alpha}+1 /|t-s|\right)\right)^{2 \alpha}}\right)^{1 / 2} \\
& =\frac{\left[c^{\phi}\left(c^{\phi} A^{\phi} \hat{q}^{2}+2 q c_{1}^{\phi} A_{1}^{\phi}\right)\right]^{1 / 2}}{\left(\ln \left(e^{\alpha}+1 /|t-s|\right)\right)^{\alpha}}, \quad 0<\alpha \leq 1 .
\end{aligned}
$$

Finally, (25) and (31) imply that

$$
\begin{aligned}
& \left(\mathrm{E}\left|X_{n, k_{j}}(t)-X_{n, k_{j}}(s)\right|^{2}\right)^{1 / 2} \\
& \quad \leq \frac{q_{1}\left[c^{\psi}\left(\hat{q}^{2} A^{\psi} c^{\psi}+2 q c_{1}^{\psi} A_{1}^{\psi}\right)\right]^{1 / 2}}{\left(\ln \left(e^{\alpha}+1 /|t-s|\right)\right)^{\alpha}}+\frac{\left[c^{\phi}\left(c^{\phi} A^{\phi} \hat{q}^{2}+2 q c_{1}^{\phi} A_{1}^{\phi}\right)\right]^{1 / 2}}{\left(\left(e^{\alpha}+1 /|t-s|\right)\right)^{\alpha}} \\
& \quad=\frac{B}{\left(\ln \left(e^{\alpha}+1 /|t-s|\right)\right)^{\alpha}}, \quad 0<\alpha \leq 1,
\end{aligned}
$$

where

$$
B=q_{1}\left[c^{\psi}\left(\hat{q}^{2} A^{\psi} c^{\psi}+2 q A_{1}^{\psi} c_{1}^{\psi}\right)\right]^{1 / 2}+\left[c^{\phi}\left(\hat{q}^{2} A^{\phi} c^{\phi}+2 q c_{1}^{\phi} A_{1}^{\phi}\right)\right]^{1 / 2} .
$$

To complete the proof, we use Theorem 2.1, where $X_{n, k_{j}}(t) \rightarrow X(t)$ in the mean square sense as $n \rightarrow \infty$ and $k_{j} \rightarrow \infty$ for $j=0,1, \ldots$. Then relation (32), Theorem 3.2, and Lemma 3.1 imply that $X_{n, k_{j}}(t) \rightarrow X(t)$ uniformly in probability in the interval $[0, T]$ as $n \rightarrow \infty$ and $k_{j} \rightarrow \infty$ for $j=0,1, \ldots$

\section{Concluding Remarks}

We have obtained sufficient conditions for the uniform convergence in probability in an interval $[0, T]$ of wavelet decompositions of strictly Orlicz stochastic processes of exponential type. We plan to apply these results for studying the rate of convergence of wavelet decompositions of strictly Orlicz stochastic processes.

\section{BIBLIOGRAPHY}

1. V. V. Buldygin and Yu. V. Kozachenko, Metric Characterization of Random Variables and Random Processes, TViMS, Kiev, 1998; English transl., American Mathematical Society, Providence, RI, 2000. MR.1743716 (2001g:60089)

2. V. V. Buldygin and Yu. V. Kozachenko, Sub-Gaussian random variables, Ukrain. Mat. Zh. 32 (1980), no. 6, 723-730; English transl. in Ukrainian Math. J. 32 (1981), no. 6, 483-489. MR598605 (82d:60023)

3. C. K. Chui, An Introduction to Wavelets, Academic Press, New York, 1992. MR.1150048 (93f:42055)

4. I. Daubechies, Ten Lectures on Wavelets, Society for Industrial and Applied Mathematics, Philadelphia, 1992. MR1162107 (93e:42045)

5. P. Flandrin, Time-Frequency/Time-Scale Analysis, Academic Press, New York, 1999. MR.1681043 (2000e:94014)

6. W. Härdle, G. Kerkyacharian, D. Picard, and A. Tsybakov, Wavelet. Approximation and Statistical Applications, Springer, New York, 1998. MR1618204 (99f:42065)

7. Yu. V. Kozachenko and O. V. Polosmak, Uniform convergence in probability of wavelet expansions of random processes from $L_{2}(\Omega)$, Random Oper. Stoch. Equ. 16 (2008), no. 4, 12-37. MR2494935 (2010c:42081) 
8. Yu. V. Kozachenko, M. M. Perestyuk, and O. I. Vasylyk, On uniform convergence of wavelet expansion of $\phi$-sub-Gaussian random processes, Random Oper. Stoch. Equ. 14 (2006), no. 3, 209-233. MR 2264363 (2008e:60092)

9. Yu. V. Kozachenko, Lectures on Wavelet Analysis, TViMS, Kyiv, 2004. (Ukrainian)

10. Yu. V. Kozachenko and G. I. Slivka, Justification of the Fourier method for hyperbolic equations with random initial conditions, Teor. Imovir. Mat. Stat. 69 (2003), 63-78; English transl. in Theory Probab. Math. Statist. 69 (2004), 67-83. MR.2110906 (2005k:60127)

11. Yu. V. Kozachenko and M. M. Perestyuk, On the uniform convergence of wavelet expansions of random processes from Orlicz spaces of random variables. I, Ukrain. Mat. Zh. 59 (2007), no. 12, 1647-1660; English transl. in Ukrainian Math. J. 59 (2007), no. 12, 1850-1869. MR2411593 (2009b:60058)

12. Yu. V. Kozachenko and M. M. Perestyuk, On the uniform convergence of wavelet expansions of random processes from Orlicz spaces of random variables. II, Ukrain. Mat. Zh. 60 (2008), no. 6, 759-775; English transl. in Ukrainian Math. J. 60 (2008), no. 6, 876-900. MR2485176 (2009m:60095)

13. Yu. V. Kozachenko and E. V. Turchin, Conditions for the uniform convergence of distributions of $\phi$-sub-Gaussian random processes in systems of functions generated by wavelets, Teor. Ĭmovir. Mat. Stat. 78 (2008), 74-85; English transl. in Theory Probab. Math. Statist. 78 (2009), 83-95. MR2446851 (2009m:60085)

14. E. Barrasa de la Krus and Yu. V. Kozachenko, Boundary-value problems for equations of mathematical physics with stricly Orlicz random initial conditions, Random Oper. Stoch. Equ. 3 (1995), no. 3, 201-220. MR.1354813 (96h:60009)

Department of Probability Theory, Statistics, and Actuarial Mathematics, Faculty for Mechanics and Mathematics, National Taras Shevchenko University, Academician Glushkov Avenue 2, Kiev 03127, Ukraine

E-mail address: yvk@univ.kiev.ua

Department of Probability Theory, Statistics, and Actuarial Mathematics, Faculty for Mechanics and Mathematics, National Taras Shevchenko University, Academician Glushkov Avenue 2, Kiev 03127, Ukraine

E-mail address: olgapolosmak@yandex.ru

Received 11/MAR/2009

Translated by O. KLESOV 